\title{
Development and psychometric properties of a pain-related problem list for adolescents (PPL)
}

\author{
Sara Weel $^{\mathrm{a}}$, Vivian Merlijn ${ }^{\mathrm{a}, \mathrm{b}, *}$, Jan Passchier ${ }^{\mathrm{a}}$, Bart Koes ${ }^{\mathrm{b}}$, Johannes van der Wouden ${ }^{\mathrm{b}}$, \\ Lisette van Suijlekom-Smit ${ }^{\mathrm{c}}$, Joke Hunfeld ${ }^{\mathrm{a}}$ \\ ${ }^{a}$ Department of Medical Psychology and Psychotherapy, Erasmus MC-University Medical Center Rotterdam, \\ P.O. Box 1738, 3000 DR Rotterdam, The Netherlands \\ ${ }^{\mathrm{b}}$ Department of General Practice, Erasmus MC-University Medical Center Rotterdam, \\ P.O. Box 1738, 3000 DR Rotterdam, The Netherlands \\ 'Department of Paediatrics, Erasmus MC Rotterdam-Sophia Children's Hospital, Rotterdam, The Netherlands
}

Received 24 October 2003; received in revised form 5 August 2004; accepted 23 August 2004

\begin{abstract}
Instruments for measuring pain-related problems in adolescents with chronic pain are sparse, especially those based on the personal experiences of these adolescents. This study aimed to develop and test such an instrument, the pain-related problem list for adolescents (PPL). A sample of 129 adolescents with chronic pain without documented physiological etiology completed the 57-item problem list, which was based on interviews with a similar group of adolescents with chronic pain. Principal components analysis yielded four domains: problems related to (1) concentration; (2) mobility; (3) adaptability; and (4) mood. The questionnaire was shortened to 18 items and has good reliability (total $\alpha=0.82$; concentration $\alpha=0.86$; mobility $\alpha=0.77$; adaptability $\alpha=0.71$; and mood $\alpha=0.78$ ); the validity also proved to be adequate, especially in the general population sample. The PPL provides a tool to assess the impact of chronic pain in adolescents. Future research should focus on further validation of the PPL in a large clinical population and establishing its test-retest reliability.
\end{abstract}

(C) 2004 Elsevier Ireland Ltd. All rights reserved.

Keywords: Pain problems; Adolescents; Chronic pain; Questionnaire development

\section{Introduction}

More than one third of Dutch adolescents aged 12-18 years suffer from chronic pain [1]. The most frequently occurring chronic pains in adolescence are headache (19\%), limb pain (16\%), abdominal pain (13\%) and back pain (11\%) [1]. Due to the high prevalence of chronic pain much research has focused on its impact on adolescents, and it has been shown that adolescents with chronic pain report lower quality of life scores than those without pain [2]. A positive relation between chronic pain and depression [3,4], and anxiety [4] has been found. Of all chronic pain types in adolescence, headache is the most extensively studied and has been shown to have a negative impact on quality of life

\footnotetext{
* Corresponding author. Tel.: +31 10 4087807; fax: +31 104089420 .

E-mail address: v.merlijn@erasmusmc.nl (V. Merlijn).
}

[5,6]. Adolescents with frequent headaches show higher levels of anxiety and depressive symptoms [7,8], and functional disability [7]. The impact of other types of chronic pain has also been studied. For example, in a sample of schoolchildren with low back pain, $94 \%$ reported some disability, mostly difficulty carrying school bags [8]. Recurrent abdominal pain is also reported to be associated with depression and anxiety [9]. A study of 59 children and adolescents with juvenile idiopathic arthritis showed a negative relationship between pain levels and the children's physical, emotional and social functioning [10]. Although chronic pain in adolescents has great impact on various aspects of quality of life and functioning, few instruments are available to measure the pain-related problems irrespective of pain localisation. Moreover, to measure specific burden associated with chronic pain, specific items based on experiences of adolescents with pain are needed. 
Such a questionnaire enables treatment planning and evaluation in clinical practice. An useful theoretical starting-point and a well-written procedure to develop our questionnaire is postulated by McKenna and co-workers $[11,12]$, we followed their procedure. They assume the needs-based model of Maslow, in which quality of life is considered the extent to which an individual is able to meet his or her needs. The development of disease-specific instruments should therefore focus on the extent to which needs are hampered by the disease in question. The problems that patients encounter when they try to fulfil their needs can determine the reduction in their quality of life. These problems differ between patients with different diseases. For instance, walking is impeded in patients with claudicatio intermittens, while thinking is obstructed in those with a migraine attack. This theoretical basis for the development of a disease-specific instrument requires the involvement of patients in the item construction both as experienced experts and as providers of the right wording of the items. The purpose of this study was to develop an interview-based list of pain-related problems that hamper the needs of adolescents with chronic pain, the pain-related problem list for adolescents (PPL), and to assess its psychometric properties.

\section{Method}

\subsection{Subjects}

Three different samples were used for this study. The first sample that was used for item construction consisted of 24 adolescents (aged 12-18 years) with chronic pain at different locations who had participated in a prevalence study [1]. These adolescents were followed-up three years later (aged 15-21 years) and interviewed about the impact of the pain on their everyday life [13]. The interviews were performed by a psychologist at the subject's home. The questions addressed pain and pain-related consequences for everyday life, particularly in terms of how the pain hampered them in their emotional, social and physical functioning, home and school activities, hobbies, going out and self-esteem. The semi-structured interviews were audiotaped and later transcribed. The content of the interviews was analysed by comparing all the consequences reported by the subjects and clustering them if they referred to the same topic. In order to maintain the patient-based nature of the instrument, the items were formulated in the actual words of the respondents, as much as possible. The interviews were examined for phrases that might be suitable for inclusion in a pain-related problems measure. According to the needs model, phrases were selected if they described the negative impact of pain on the adolescent's ability to meet their needs. This was done by a team consisting of three psychologists, a pedagogue and a paediatrician, leading to the formulation of 57 items. Before distributing the pain- related problem items at school, the items were presented to five adolescent girls without chronic pain to test their face validity.

After determining the face validity, a second sample of 447 students from a secondary school in Rotterdam was used for item reduction and validation. The adolescents were asked to complete the pain-related problem items and additional questionnaires during class. The questionnaires were administered to students of all years and educational levels. Students were included in the study sample if they were 12-18 years, and experienced recurrent or continuous pain (without a known organic cause), existing for three months or longer. As the PPL addresses the impact of the pain in the previous week (the week before administration), students were excluded if they had no pain in the previous week, or if the pain of the previous week was different from the location of their chronic pain.

The goal of the study (i.e., to develop a pain-related problem list for adolescents with chronic pain) was explained and instructions on how to fill out the questionnaires were given by one of the researchers. The researcher and the teacher remained in the class during completion.

The questionnaires were also administered to a clinical sample for further validation. The sample consisted of 31 adolescents (12-18 years) with chronic pain with no organic aetiology who were enrolled for a psychosocial intervention on learning how to cope with chronic pain in the Erasmus University Medical Center.

\subsection{Measures}

\subsubsection{Pain-related problems list}

The response categories of the 57 pain-related problems ranged from 0 (seldom or never) to 3 (very often or always).

\subsubsection{Demographic data}

Demographic data included the adolescent's date of birth, gender, nationality, educational level and current school year.

\subsubsection{Pain}

The Pain questionnaire [1] collected information about the location, frequency, duration of the pain episodes and history (i.e., number of months in pain) using a retrospective format. From a list of possible locations (head, abdomen, back, limb, neck, ear, throat, chest and elsewhere) subjects were asked to indicate all locations where they had experienced recurrent or continuous chronic pain in the previous three months. Pain intensity was measured with the chronic pain disability inventory (CPDI). Adolescents were asked whether they had consulted a physician or general practitioner about their pain and, if so, the physician's diagnosis. 


\subsubsection{Additional questionnaires}

- CPDI: The chronic pain disability inventory is a sevenitem questionnaire, which measures the intensity of the pain and disability caused by pain in the previous month. It consists of three pain intensity items and four disability items. Response categories range from 0 ("no pain" or "no disability") to 10 ("worst imaginable pain" or "complete disability"). The CPDI was adapted from the chronic pain grading scale, which is a reliable and valid instrument [14].

- PedMIDAS: The pediatric migraine disability assessment (PedMIDAS) [15] is a six-item questionnaire that assesses disability caused by headache in children and adolescents. For each item, subjects report the number of days a particular aspect of functioning was impaired in the previous three months due to headache. It has shown to be a sensitive, reliable and valid instrument for this group of patients [15]. The items in PedMIDAS have been translated by us into Dutch and adapted to suit all pain locations by replacing the word "headache" with the word "pain".

- $Q L A-C P$ : Two domains of the quality of life questionnaire for adolescents with chronic pain (QLA-CP) [16], measuring satisfaction with life in general and satisfaction with health, were included for validation in the general population sample. Both domains were measured with a visual analogue scale (VAS). This is a $100-\mathrm{mm}$ line with the anchors "completely dissatisfied" (0 mm) to "completely satisfied" (100 mm). In addition, the four remaining domains (psychological functioning, functional status, physical functioning and social functioning) were administered for validation in the clinical sample. The QLA-CP has shown suitable consistency and construct validity against COOP/WONCA charts [16]. A higher score on each domain of the QLA-CP represents a better quality of life.

\subsection{Data analysis}

To identify the internal structure of the PPL and to reduce the number of items a principal components analysis was conducted with varimax rotation. It was first set to extract the components with an eigenvalue $\geq 1$. Subsequently, a scree plot of the eigenvalues was used to determine the number of components to retain. Items with a component loading $<0.40$ were excluded [17]. Additional reduction was done by excluding items that contributed little to the internal consistency of that specific component.

Domain scores were computed by averaging the items loading on each component. A total score was obtained by summing the domain scores.

Internal consistency of the PPL total score and its domains were evaluated by Cronbach's coefficient alpha. Pearson's correlations were carried out between the PPL with the disability items of the PedMIDAS items and the CPDI, the two visual analogue scales of the QLA-CP, and the pain parameters (i.e., frequency and intensity of the pain). To assess the validity of the PPL in a clinical practice, Pearson's correlations were also calculated with the CPDI, all domains of the QLA-CP and with pain parameters. Correlations between similar domains and items were expected to be strong, thereby indicating convergent validity. Correlations between dissimilar domains and items were expected to be weaker and reflect divergent validity. Convergent validity was also tested in both the general and clinical sample by comparing the pain-related problem scores of adolescents with high pain intensity with those with low intensity using independent $t$-tests. A score higher than the median (for both samples 5 on a scale of 0 'no pain' to 10 'worst imaginable pain') was considered to be high intensity, low intensity was a score of 5 or lower.

Table 1

Characteristics of the study population

\begin{tabular}{lcr}
\hline & General population $(n=129)^{\mathrm{a}}$ & Clinical sample $(n=31)$ \\
\hline Demographic factors & & \\
Age: mean in years (S.D.) & $15.1(1.6)$ & $15.2(2.0)$ \\
Gender: number (\%) & & $2(0.7)$ \\
$\quad$ Boys & $37(28.7)$ & $29(93.0)$ \\
$\quad$ Girls & $90(69.8)$ & \\
Pain characteristics & & $5.3(2.2)$ \\
Intensity: mean (S.D.) & & \\
Location: number (\%) & & \\
Head & & \\
Limb & $39(30.2)$ & $14(45.0)$ \\
Back & $25(19.4)$ & $5(16.0)$ \\
Abdomen & $17(13.2)$ & $4(13.0)$ \\
Neck & $15(11.6)$ & $4(13.0)$ \\
Multiple & $6(4.7)$ & - \\
Other & $20(15.5)$ & - \\
\hline
\end{tabular}

\footnotetext{
${ }^{\text {a }}$ Gender of 2 subjects missing.

b Range of scores $0-10$.

c Pain location that troubled the adolescents the most.
} 


\section{Results}

\subsection{Subjects}

The mean age of the 24 subjects included in the sample for item construction was 17.5 years, four of whom were male $(16.7 \%)$ and $20(83.3 \%)$ female. Pain was reported in the head $(54.2 \%)$, limbs and/or back $(41.2 \%)$ as well as multiple pains (abdomen and limbs, 4.2\%). The five adolescent girls without chronic pain participating in the face validity study had a mean age of 15 years (range 13-18 years).

Table 1 gives the characteristics of the 129 subjects (aged 12-18 years) included in the sample for item reduction and validation of the PPL. The majority were girls $(71 \%)$ and the most frequently reported pain was headache $(30 \%)$. The mean pain intensity was 5.2 $($ S.D. $=2.2)$ on a scale ranging from 0 to 10 . On average pain was experienced once a week. The pain existed on average for 29.2 months (S.D. = 29.9).

Table 1 also shows the characteristics of the 31 subjects (aged 12-18 years) in the clinical sample. The majority were girls $(93 \%)$ and the most frequently reported pain was headache $(45 \%)$. The mean pain intensity was 5.3 $($ S.D. $=2.2)$, on a scale ranging from 0 to 10 . The majority $(66 \%)$ of the adolescents experienced their pain every day. The pain existed on average for 47.5 months (S.D. = 23.3).

\subsection{Item construction}

After content analysis of the interviews, 57 items concerning the impact of pain were formulated. Response categories for the 57 items ranged from 0 (seldom or never) to 3 (very often or always). Considering the face validity of the 57 items none of the five adolescents had difficulty understanding the items; all attributed the same meaning to the items as intended by the researchers. The only change made to the items was the addition of an example ('a schoolbag') in the item "the pain troubled me when lifting'.

\subsection{Item reduction and validation}

Principal components analysis extracted 16 components with an eigenvalue $>1$. Examination of the scree plot suggested four components; this was also the solution that was best interpretable. The four components together explained $42 \%$ of the variance; these components were designated: problems related to concentration, mobility, adaptability and mood.

Of the 57 items, five had a loading $<0.40$ on each of the four components, and these were excluded from further analyses. Supplementary item reduction was done by examining the internal consistency of each of the components. Items contributing little or nothing to the internal consistency were also excluded. Our purpose was to

Table 2

Component loadings and internal consistency of the pain-related problem list

\begin{tabular}{|c|c|c|c|}
\hline Components and items & Component loadings & Cronbach's alpha & Scores $^{\mathrm{a}} M(\mathrm{SD})$ \\
\hline \multicolumn{4}{|l|}{ Concentration } \\
\hline 1. Drowsiness & 0.71 & 0.86 & $2.14(0.82)$ \\
\hline 2. Trouble with reading & 0.71 & & \\
\hline 3. Prolonged mental activity & 0.75 & & \\
\hline 4. Trouble with thinking & 0.71 & & \\
\hline Mobility & & 0.77 & $2.40(0.58)$ \\
\hline 5. Unable to play sports & 0.69 & & \\
\hline 6. Trouble with walking & 0.46 & & \\
\hline 7. Trouble with pushing & 0.56 & & \\
\hline 8. Trouble with lifting & 0.62 & & \\
\hline 9. Trouble with carrying & 0.60 & & \\
\hline Adaptability & & 0.71 & $2.72(0.44)$ \\
\hline 10. Must wear flat shoes & 0.68 & & \\
\hline 11. Use of aids at school/work & 0.70 & & \\
\hline 12. Frequent toilet use & 0.49 & & \\
\hline 13. Can not eat much & 0.63 & & \\
\hline 14. Must sit bent over & 0.59 & & \\
\hline Mood & & 0.78 & $1.83(0.69)$ \\
\hline 15. Feel peevish & 0.54 & & \\
\hline 16. Have bad moods & 0.56 & & \\
\hline 17. React angrily to others & 0.59 & & \\
\hline 18. Feel like doing nothing & 0.59 & & \\
\hline Total & & 0.82 & $9.10(1.69)$ \\
\hline
\end{tabular}

Note: range of subscale scores: 0-3, and range of total: 0-12.

${ }^{a}$ Higher score indicates more pain-related problems. 
retain a Cronbach's alpha of at least 0.70 for each component [17]. In this way, the original 57 items were reduced to 18 items.

The internal consistency (Cronbach's alpha) was adequate for the domains and the total score. Table 2 lists the 18 remaining items, the factor loadings, Cronbach's alpha and pain-related problem scores. These items together formed the PPL (pain-related problem list for adolescents).

Table 3 gives the Pearson product-moment correlations of the PPL total score and domain scores, with pain intensity (CPDI items) and pain frequency. Problems in concentration, mobility and adaptability, and the total scale show significant positive correlations with pain intensity, indicating that adolescents with higher pain intensity experienced more problems in these areas. This was also shown by $t$-tests comparing adolescents with low pain intensity (score $\leq 5$ ) to adolescents with high pain intensity (score $>5$ ). Adolescents with high pain intensity scored higher on problems in concentration $(t(112.9)=-2.33, P<0.05)$, mobility $(t(126)=-6.19, P<0.001)$, adaptability $(t(125)=$ $-2.89, P<0.01)$ and the total scale $(t(123)=-4.42$, $P<0.001)$ than adolescents with low pain intensity. Mood was the only domain that was less affected by the pain intensity, i.e., adolescents with high and low pain intensity did not report much differences in problems related to their mood. Mobility is the only domain significantly correlated with pain frequency, indicating that adolescents with more frequent pain are less mobile.

Table 3 also shows the correlations between the PPL and satisfaction with life and health, CPDI disability items and
PedMIDAS items. The problems related to concentration showed significant positive correlations with items concerning schoolwork, daily functioning and negative correlations with quality of life. Problems related to the mobility correlated with all disability items of the CPDI and items on diminished functioning of the PedMIDAS. Problems related to adaptability showed significant positive correlations with items on normal and fun activities of the CPDI and satisfaction with health, and mood had a moderate correlation with the two satisfaction scales, items related to school and normal activity of the CPDI. Finally, the total scale showed significant correlations with most items.

Table 4 gives the Pearson product-moment correlations of the PPL total score and domain scores, with the pain intensity items of the CPDI and pain frequency. Problems related to mobility and adaptability show significant positive correlations with pain intensity indicating that adolescents with higher pain intensity experienced more problems in these areas. This was also shown by $t$-tests comparing adolescents with low pain intensity (score $\leq 5$ ) to adolescents with high pain intensity (score $>5$ ). Adolescents with high pain intensity scored higher on the total scale $(t(28)=-2.15, P<0.05)$ and on problems related to the adaptability $(t(28)=-2.36, P<0.05)$ than adolescents with low pain intensity. Although not significant, adolescents with a higher pain intensity tended to report more problems related to mobility $(t(29)=-1.77, P=0.08)$. As for the general population, problems related to mobility are significantly correlated to pain frequency. Table 4 also shows the correlations between the PPL with the disability items of

Table 3

Pearson correlations between the PAQoL-A total and domains, and between pain parameters, satisfaction with life and health, CPDI and PedMIDAS

\begin{tabular}{|c|c|c|c|c|c|}
\hline & Concentration & Mobility & Adaptability & Mood & Total \\
\hline \multicolumn{6}{|l|}{ Pain } \\
\hline Pain frequency & 0.03 & $0.28^{* *}$ & -0.03 & -0.03 & 0.08 \\
\hline \multicolumn{6}{|l|}{ Pain items CPDI } \\
\hline 1. How worse is the pain now? & $0.37^{* *}$ & $0.43^{* *}$ & $0.24^{* *}$ & $0.21^{*}$ & $0.48^{* *}$ \\
\hline 2. How worse is the pain usually in the past month? & $0.31^{* *}$ & $0.47^{* *}$ & $0.33^{* *}$ & 0.14 & $0.48^{* *}$ \\
\hline 3. How was the most awful pain in the past month? & $0.27^{* *}$ & $0.37^{* *}$ & $0.24^{* *}$ & $0.15^{\mathrm{T}}$ & $0.41^{* *}$ \\
\hline \multicolumn{6}{|l|}{ Disability items CPDI } \\
\hline 4. Days unable to do (school) work & $0.37^{* *}$ & $0.20^{*}$ & $0.19^{*}$ & $0.29^{* *}$ & $0.41^{* *}$ \\
\hline 5. (School) Work burdened by pain & $0.43^{* *}$ & $0.33^{* *}$ & 0.17 & $0.21^{*}$ & $0.45^{* *}$ \\
\hline 6. Normal activities burdened by pain & $0.32^{* *}$ & $0.53^{* *}$ & $0.37^{* *}$ & $0.20^{*}$ & $0.54^{* *}$ \\
\hline 7. Fun activities burdened by pain & $0.29^{* *}$ & $0.47^{* *}$ & $0.23^{*}$ & 0.13 & $0.43^{* *}$ \\
\hline \multicolumn{6}{|l|}{ PedMIDAS items } \\
\hline 1. Schooldays missed & $0.30^{* *}$ & 0.06 & 0.04 & 0.16 & $0.24^{* *}$ \\
\hline 2. Partial schooldays missed & 0.15 & $0.23^{* *}$ & 0.06 & 0.08 & $0.21^{* *}$ \\
\hline 3. Days functioned less than normal & $0.34^{* *}$ & $0.19^{*}$ & 0.16 & 0.10 & $0.31^{* *}$ \\
\hline 4. Days not able to do anything at home & $0.25^{* *}$ & 0.02 & 0.06 & 0.14 & $0.20^{*}$ \\
\hline 5. Days not able to join activities after school & 0.06 & 0.07 & .01 & -0.02 & 0.05 \\
\hline 6. Days joined in activities while functioning less than normal & 0.16 & $0.30^{* *}$ & 0.15 & 0.01 & $0.23^{*}$ \\
\hline \multicolumn{6}{|l|}{ Quality of life } \\
\hline Satisfaction with life & $-0.35^{* *}$ & -0.06 & -0.17 & $-0.46^{* *}$ & $-0.42^{* *}$ \\
\hline Satisfaction with health & $-0.49^{* *}$ & -0.09 & $-0.19^{*}$ & $-0.41^{* *}$ & $-0.48^{* *}$ \\
\hline
\end{tabular}

${ }^{*} P<0.05$.

** $P<0.01$.

${ }^{\mathrm{T}}$ Did not reach significance level of 0.05 . Indicates a tendency-level. 
Table 4

Pearson correlations between the pain-related problem list, including the total score and the domains with the pain parameters, the domains of quality of life (QLA-CP) and disability (CPDI) in the clinical sample

\begin{tabular}{|c|c|c|c|c|c|}
\hline & Concentration & Mobility & Adaptability & Mood & Total \\
\hline \multicolumn{6}{|l|}{ Pain } \\
\hline Pain frequency & 0.05 & $0.47^{* *}$ & 0.19 & 0.03 & 0.29 \\
\hline \multicolumn{6}{|l|}{ Pain items CPDI } \\
\hline 1. How worse is the pain now? & 0.09 & 0.22 & 0.05 & 0.05 & 0.16 \\
\hline 2. How worse is the pain usually in the past month? & 0.02 & $0.36^{*}$ & $0.42^{*}$ & 0.17 & $0.32^{\mathrm{T}}$ \\
\hline 3. How was the most awful pain in the past month? & 0.22 & $0.48^{* *}$ & 0.21 & 0.28 & $0.45^{*}$ \\
\hline \multicolumn{6}{|l|}{ Disability items CPDI } \\
\hline 4. Days unable to do (school) work & 0.02 & $0.36^{*}$ & 0.25 & -0.14 & 0.09 \\
\hline 5. (School) Work burdened by pain & $0.41^{*}$ & $0.57^{* *}$ & 0.30 & 0.29 & $0.59^{* *}$ \\
\hline 6. Normal activities burdened by pain & $0.35^{*}$ & $0.45^{*}$ & 0.25 & 0.22 & $0.49^{* *}$ \\
\hline 7. Fun activities burdened by pain & 0.04 & $0.46^{* *}$ & $0.46^{* *}$ & 0.01 & 0.29 \\
\hline \multicolumn{6}{|l|}{ Quality of life } \\
\hline Satisfaction with life & $-0.38^{*}$ & 0.15 & 0.04 & -0.18 & -0.15 \\
\hline Satisfaction with health & -0.15 & $-0.52^{* *}$ & -0.08 & 0.08 & -0.27 \\
\hline Psychological functioning & $-0.42^{*}$ & -0.05 & -0.03 & $-0.41^{*}$ & $-0.35^{*}$ \\
\hline Functional status & $-0.49^{* *}$ & $-0.50^{* *}$ & -0.12 & -0.13 & $-0.50^{* *}$ \\
\hline Physical functioning & -0.27 & 0.15 & -0.24 & -0.23 & -0.21 \\
\hline Social functioning & -0.22 & 0.05 & -0.01 & -0.17 & -0.09 \\
\hline
\end{tabular}

the CPDI and the QLA-CP. The problems related to concentration showed moderate correlations with items concerning schoolwork and normal activities while, as expected, mobility correlated strongly with all CPDI items. Problems in adaptability only correlated with items on fun activities, but mood-related problems did not correlate with any of the CPDI-items. The total score correlated highly with items related to schoolwork and normal activities. The problems related to concentration showed negative correlations with psychological functioning, satisfaction with life and functional status of the adolescents, indicating that more pain-related problems are related to a lower quality of life. The problems related to mobility correlated high but negatively with satisfaction with health and the functional status, while mood-related problems only correlated negatively with the psychological functioning of the adolescents. Finally, the total scale showed strong and negative correlations with the functional status of the adolescent (in terms of impact of the pain on daily activities and leisure activities).

\section{Discussion and conclusion}

\subsection{Discussion}

This study was designed to develop a pain-related problem list for adolescents with chronic pain. A principal component analysis was performed and the internal consistency and validity of the instrument were established. This resulted in the pain-related problem list, an 18-item questionnaire consisting of four domains: problems with (1) concentration, (2) mobility, (3) adaptability, and (4) mood.
In the general population sample, the total scale and all the domains except problems related to mood correlated negatively and significantly with pain intensity. These results might indicate that negative mood in adolescents is related more to other factors (e.g., developmental tasks [18] or vulnerability [19]) than to pain. However, this suggestion needs further research.

In this study, we used the CPDI and the PedMIDAS to examine the PPL in a general population. One limitation of our study could be that the PedMIDAS may not have been an ideal instrument with which to validate our scale. The PedMIDAS originally was validated in patients from a tertiary referral center for paediatric headaches, whereas the PPL is based on experiences of adolescents in the general population and with chronic pain in various locations. However, at present, few validated instruments are available, which are short and easy to administer, and measure painrelated problems of adolescents with chronic pain.

One of the main methodological strengths of the present study is the use of interviews to construct the items. The items thus reflect the experiences of adolescents with chronic pain and their related problems rather than the opinion of professionals or exclusively theoretical constructs. Another strength is the applicability of our questionnaire to pain in different locations as opposed to only one pain location, which is the case with most other pain-related questionnaires on daily functioning [20,21].

Further study is necessary on the test-retest reliability and responsiveness of this instrument. Because the PPL is a Dutch-language questionnaire, cross-cultural validation should be established. 


\subsection{Conclusion}

The internal consistency of the domains and the total scale of the PPL proved to be adequate. We also found evidence for the validity of the total scale and the four domains in the general population. Some indication was found for validation in a clinical sample.

The total scale and the four domains all showed convergent and divergent validity in both samples. They had the strongest correlations with the items we expected, and the other items had either a weaker correlations or were not significant. This confirms our hypothesis that the PPL has adequate validity. Compared to the general population, we found less but stronger correlations in the clinical sample, which might be caused by the small number of patients. Therefore, a further validation of the PPL in a larger clinical sample is needed.

\subsection{Practice implications}

This relatively short questionnaire (18 items) can easily be implemented in routine clinical practice and enables the assessment of the impact of chronic pain on adolescents with different types of chronic pain. Because the items are based on the experiences of adolescents with chronic pain, the PPL reflects pain-related problems that hamper the needs of these adolescents. When complemented with a generic quality of life instrument, like the QLA-CP or child health questionnaire (CHQ), health care providers are able to obtain a complete picture on the impact of chronic pain on adolescents in terms of problems and quality of life. In addition, the PPL can be used to evaluate the provided (health) care in the course of time. Finally, the answers given by an adolescent with pain to the individual items can help the clinician to identify the main problems on which treatment should focus. Consequently, the PPL is, additional to being a useful tool in pain research, a useful supplement for assessment in clinical practice.

\section{Acknowledgements}

We wish to thank the 'Algesiology Foundation' for their financial support and Wieke Bertina for her contribution to the item construction.

\section{References}

[1] Perquin CW, Hazebroek-Kampschreur AAJM, Hunfeld JAM, Bohnen AM, van Suijlekom-Smit LWA, Passchier J, et al. Pain in children and adolescents: a common experience. Pain 2000;87:51-8.
[2] Merlijn VPBM, Hunfeld JAM, van der Wouden JC, HazebroekKampschreur AAJM, Koes BW, Passchier J. Psychosocial factors associated with chronic benign pain in adolescents. Pain 2003;101: $33-43$.

[3] Harma AM, Kaltiala-Heino R, Rimpela M, Rantanen P. Are adolescents with frequent pain symptoms more depressed? Scand J Prim Health Care 2002;20:92-6.

[4] Varni JW, Rapoff MA, Waldron SA, Gragg RA, Bernstein BH, Lindsley CB. Chronic pain and emotional distress in children and adolescents. J Dev Behav Pediatr 1996;17:154-61.

[5] Bandell-Hoekstra IE, Abu-Saad HH, Passchier J, Frederiks CM, Feron FJ, Knipschild P. Coping and quality of life in relation to headache in Dutch schoolchildren. Eur J Pain 2002;6:315-21.

[6] Nodari E, Battistella PA, Naccarella C, Vidi M. Quality of life in young Italian patients with primary headache. Headache 2002;42: 268-74.

[7] Fichtel $\AA$, Larsson B. Psychosocial impact of headache and comorbidity with other pains among Swedish school adolescents. Headache 2002;42:766-75.

[8] Watson KD, Papageorgiou AC, Jones GT, Taylor S, Symmons DPM, Silman AJ, et al. Low back pain in schoolchildren: occurrence and characteristics. Pain 2002;97:87-92.

[9] Liakopoulou-Kairis M, Alifieraki T, Protagora D, Korpa T, Kondyli K, Dimosthenous E, et al. Recurrent abdominal pain and headache. Psychopathology, life events and family functioning. Eur Child Adolesc Psychiatry 2002;11:115-22.

[10] Sawyer MG, Whitham JN, Roberton DM, Taplin JE, Varni JW, Baghurst PA. The relationship between health-related quality of life, pain and coping strategies in juvenile idiopathic arthritis. Rheumatology 2004;43:325-30.

[11] McKenna S, Patrick D. Development of a migraine specific quality of life measure for multi-national clinical trials. Cephalalgia 1995;15: 280

[12] Hunt SM, McKenna SP. The QLDS: a scale for the measurement of quality of life in depression. Health Policy 1992;22:307-19.

[13] Hunfeld JAM, Perquin CW, Bertina W, Hazebroek-Kampschreur AAJM, van Suijlekom-Smit LWA, Koes BW, et al. Stability of pain parameters and pain-related quality of life in adolescents with persistent pain: a three-year follow-up. Clin J Pain 2002;18:99-106.

[14] Von Korff M, Ormel J, Keefe FJ, Dworkin SF. Grading the severity of chronic pain. Pain 1992;50:133-49.

[15] Hershey AD, Powers S, Vockell ALB, LeCates S, Kabbouche MA, Maynard MK. PedMIDAS. Development of a questionnaire to assess disability of migraines in children. Neurology 2001;57:2034-9.

[16] Merlijn VPBM, Hunfeld JAM, van der Wouden JC, HazebroekKampschreur AAJM, Passchier J. A shortening of the QLH-Y: the quality of life questionnaire for adolescents with chronic pain (QLACP) and its psychometric qualities. Psychol Rep 2002;90:753-9.

[17] Nunnally JC, Bernstein IH. Psychometric Theory. New York: McGraw-Hill Inc.; 1994.

[18] Remschmidt H. Psychosocial milestones in normal puberty and adolescence. Horm Res 1994;41:19-29.

[19] Passchier J, de Boo M, Quaak HZ, Brienen JA. Health-related quality of life of chronic headache patients is predicted by the emotional component of their pain. Headache 1996;36:556-60.

[20] Hartmaier SL, DeMuro-Mercon C, Linder S, Winner P, Santanello NC. Development of a brief 24-hour adolescent migraine functioning questionnaire. Headache 2001;41:150-6.

[21] Passchier J, Mourik JC, McKenna SP, van den Berg M, Erdman RA... Evaluation of the Dutch version of the migraine quality of life instrument (MSQOL) and its application in headache coping. Cephalalgia 2001;21:823-9. 